\title{
Detection and analysis of surface currents in western Guangdong by compact surface wave radar
}

\author{
zili $\mathrm{Li}^{1}$, and Junjie $\mathrm{Li}^{1{ }^{1 *}}$ \\ ${ }^{1}$ College of Electronic Engineering, Guangxi Normal University, Guilin 541004, China
}

\begin{abstract}
In order to study the detection performance of compact surface wave radar on ocean surface current in the west of Guangdong Province, firstly, the bistatic radar is set up to collect ocean echo data on the west coast of Guangdong Province. Then, by using the Multiple Signal Classification (MUSIC) algorithm, the comparative analysis results of ocean surface current information and the actual measurement results of DVS Current Meter are obtained. The root mean square error of flow velocity less than $10 \mathrm{~cm} / \mathrm{s}$ and the root mean square error of flow direction less than 25 degrees were obtained. It shows that compact surface wave radar can achieve good results and has good detection capability in the west of Guangdong, china.
\end{abstract}

\section{Introduction}

West Guangdong Sea, located in the southwest of the north of South China Sea, includes the sea from the west of Pearl River Estuary to the north of Hainan Island [1]. The coastal area of the west of Guangdong is a sea area closely related to the contemporary economic development, and its current state has an important influence on mariculture, material transportation and offshore engineering in this area. In order to effectively sup-port the development of Marine economy, it is necessary to carry out real-time, continuous and comprehensive monitoring of ocean surface current, and then provide accurate multi-time and multi-scale Marine environmental forecast [2].

At present, high frequency surface wave radar is the main tool capable of measuring ocean surface current state in a large range and with high precision, nearly 500 high frequency radar systems have been set up around the world[3]. These radar systems can be divided into two types in antenna form: array HF surface-wave radar based on beam-forming technology and compact HF surfacewave radar based on direction finding technology . Among them, the phased array antenna is used as the receiving antenna for the array high frequency surface wave radar represented by the WERA system (Germany). The receiving antennas of portable high frequency surface wave radars, represented by Seasonde system (USA) and OSMAR-S system (China), adopt monopole cross loops. However, the antenna array of array HF surface wave radar is relatively large, which brings the disadvantages such as large area, difficulty in erection and high power consumption[3]. However, compact portable radar does not need to specially open up a site for its antenna. It is relatively easy to locate, build and maintain the station, and has strong adaptability to installation. It is the main direction of the development of high frequency surface wave radar. In the international high frequency surface wave radar market, portable high frequency surface wave radar occupies more than $90 \%$ of the market share .

The OSMAR-S series compact high frequency surface wave radar developed by China has been tested in many sea areas to detect ocean surface currents. In this paper, the performance of the Omar-S portable radar system is tested by the current measurement data of the current meter, and the trial situation of the Omar-S portable radar system in the sea area of the west of Guangdong is studied, so as to provide a useful reference for the application of the compact surface-wave radar in the sea area.

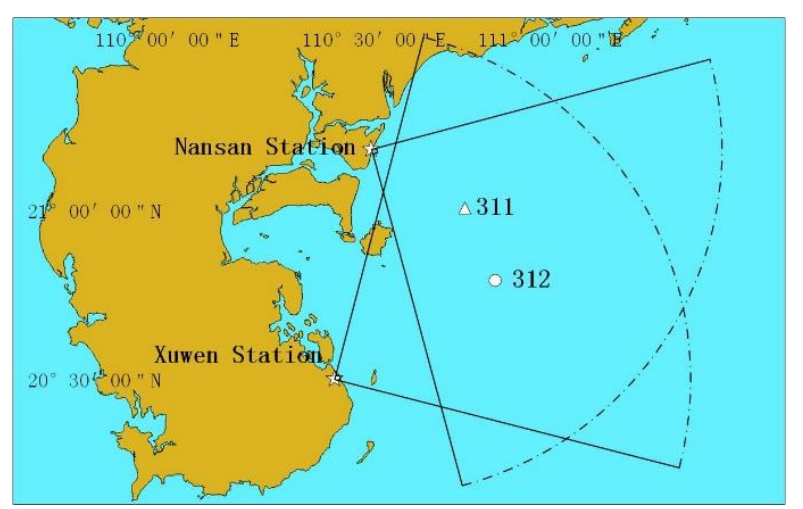

Figure 1. Location map of radar and current meter.

\section{Overview of Compact Surface Wave Radar System}

OSMAR-S is a coherent scattering radar system, which mainly consists of transmit-ting antenna, receiving antenna, receiver, transmitter and display terminal [9]. The trans-mitting antenna of the system is a whip antenna and the receiving antenna is a monopole crossed ring antenna. After the receiving signal is preprocessed, the

\footnotetext{
*Corresponding author:61419008@qq.com
} 
radial velocity of the ocean surface is calculated by combining with the system parameters. The radial flows in different directions of the same measuring point are obtained by the bistatic radar, and the vector flow field of the measured point is obtained by combining the vector synthesis method [10]. The operating frequency of the radar is $13 \pm 0.5 \mathrm{MHz}$; the maximum detection range is 100 $\mathrm{km}$; the radial range resolution is $2.5 \mathrm{~km}$; the velocity resolution is $5 \mathrm{~cm} / \mathrm{s}$; and the angular resolution is $2.5^{\circ}$.

In this experiment, the bistatic radar system was used to install the high frequency surface wave radar in Nansan Town $\left(110^{\circ} 35^{\prime} 16^{\prime \prime} \mathrm{E} 、 21^{\circ} 8^{\prime} 13^{\prime \prime} \mathrm{N}\right)$ and Xuwen County $\left(110^{\circ} 29^{\prime} 49^{\prime \prime} \mathrm{E} 、 20^{\circ} 32^{\prime} 46^{\prime \prime} \mathrm{N}\right)$ of Zhanjiang City, Guangdong Province. As shown in Figure 1, the triangle and the circle indicate the specific positions of two DVS current meters 311 and 312 , which are located at $110^{\circ} 49^{\prime} 43^{\prime \prime} \mathrm{E}, 20^{\circ} 59^{\prime} 25^{\prime \prime} \mathrm{N}$ and $110^{\circ} 53^{\prime} 48^{\prime \prime} \mathrm{E}, 20^{\circ} 47^{\prime} 40^{\prime \prime} \mathrm{N}$. The five-pointed star is the location of two radar stations, respectively named as Nansan Station (located in Nansan Town, Zhanjiang City) and Xuwen Station (located in Xuwen County, Zhanjiang City). The two fan regions are the theoretical coverage range of electromagnetic waves of the two radar stations, with a radius of $100 \mathrm{~km}$. The radar flow measurement area is the common coverage range of the two radar stations.

\section{Extraction of surface flow}

\subsection{Extraction of radial flow velocity}

In the experiment, the scattered echo signals from the ocean surface are received from the radar station according to the principle of interrupted frequency modulated continuous wave. The received echo signals are separated into different range units, and the sum and average of the echo power received by each channel in the coherent accumulation time are obtained to obtain the original echo data detected by the radar. The echo spectrum of each range element is obtained by two fast Fourier transform of the original data of a single channel, and then the spectrum of all range elements is combined to get the range Doppler spectrum of the whole detection area [5].

The velocity information of ocean current is determined by the frequency shift of first-order internal frequency points relative to Bragg frequency, so it is necessary to determine which effective frequency points can be used to retrieve ocean current. The echo in a distance element is not only the first order echo of ocean waves but also the high order echo and other noises. In order to determine the first-order peak frequency points, it is usual to define the maximum frequency offset of the first-order peak by setting a maxi-mum probed velocity, which is obtained from the historical data in the observed area. Then the noise power intensity is calculated according to the noise region. Finally, the preset threshold value of SNR $(\mathrm{dB})$ is used to select the first-order peak frequency points that meet the requirements of SNR. The process of determining the effective frequency points of the first order peak in the Doppler spectrum is shown in
Fig.2, where the SNR threshold is set at $10 \mathrm{~dB}$, and the two gray areas above the SNR threshold in the figure are the final determined first-order peak frequency points. Of course, there are other methods to select the first-order peak, such as the difference spectrum method and the first-order peak separation method based on image processing technology[13], but the method used here to select the first-order peak frequency points by setting the historical maximum flow rate and the SNR threshold is still the most convenient and the most widely used.

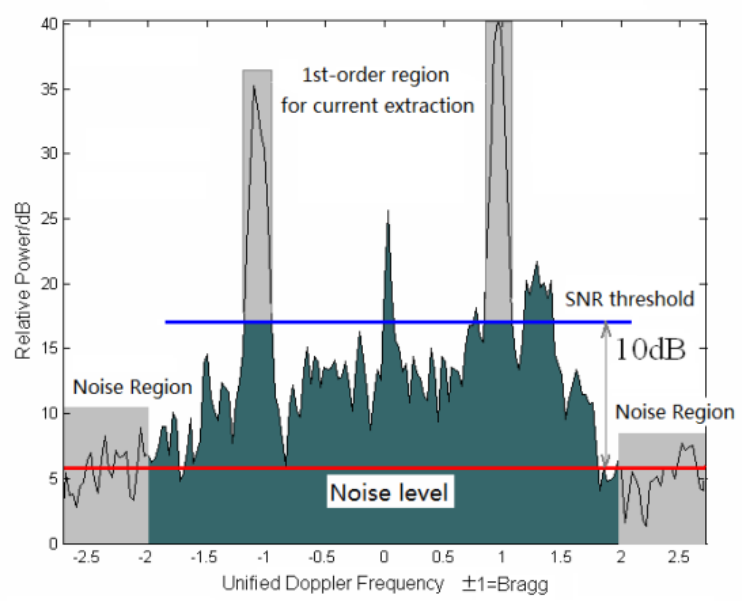

Figure 2. Schematic diagram of first order peak extraction.

After the first-order spectrum containing the ocean surface current information is obtained, the magnitude of the radial velocity can be calculated. The corresponding relationship between the frequency offset of the corresponding spectral point and the ocean surface radial velocity is as follows:

$$
\Delta f=2 v / \lambda
$$

Where is the operating wavelength of the radar. The radial velocity can be calculated by Equation (1).

\subsection{Extraction of radial flow direction}

After the size of the radial flow is obtained, the next step is to determine the radial flow orientation. Spatial spectrum estimation in array signal processing is a very popular field of study, when the signal into the antenna array, as a result of the existence of array element spacing, the antenna receives signals exist fixed phase difference, there is a phase difference is frequency, azimuth estimation became the frequency spectrum estimation, so you can use the classic spectrum estimation and modern spectrum estimation algorithm to estimate azimuth[6].

MUSIC (Multiple Signal Classification) directional algorithm has a common applicability, can deal with arbitrary shape, arbitrary direction characteristics of antenna array, can give a good result in practical application, the sea echo theory shows that the first-order sea echo Signal is Gaussian Signal, and on the time and location are quick to related, so the MUSIC algorithm is applied to the radial flow calculation. 
When the number of signal sources $\mathrm{N}$ is less than the number of antenna array elements $M$, the mathematical model of the narrow-band far-field signal is:

$$
X(t)=A(\theta) S(t)+N(t)
$$

$X$ is the antenna receiving data, $A$ is the antenna array type, and $N$ is the noise. The covariance of the received data is:

$$
R=E\left[X X^{H}\right]=A E\left[S S^{H}\right] A^{H}+\sigma^{2} I=A R_{S} A^{H}+\sigma^{2} I
$$

Where $R_{s}$ is the covariance of the signal source, $A R_{S} A^{H}$ is the signal part, and $\sigma^{2} I$ is the noise part.

The eigendecomposition of $R$ can be obtained as follows:

$$
R=U_{S} \Sigma_{S} U_{S}^{H}+U_{N} \Sigma_{N} U_{N}^{H}
$$

$U_{S}$ is the signal subspace, $U_{N}$ is the noise subspace, $\Sigma_{S}$ and $\Sigma_{N}$ are the corresponding eigenvalues of the signal and noise respectively.

Since $U_{S}$ and $U_{N}$ are orthogonal after eigendecomposition, and the span of $U_{S}$ and the guide vector $a(\theta)$ of the signal is the same space, so:

$$
a^{H}(\theta) U_{N}=0
$$

We are dealing with data of finite length, so we can only get the maximum likelihood estimate of $R$ :

$$
\hat{R}=\frac{1}{L} \sum_{i=1}^{L} X_{i} X_{i}^{H}
$$

Estimation $\hat{U}_{S}$ and $\hat{U}_{N}$ of signal subspace and noise subspace can be obtained by eigendecomposition of $\hat{R}$. Due to the presence of noise, $a(\theta)$ and $\hat{U}_{N}$ cannot be completely orthogonal, but there is a minimum value, which MUSIC algorithm determines the azimuth by searching:

$$
P(\theta)=\frac{1}{a^{H}(\theta) \hat{U}_{N} \hat{U}_{N}^{H} a(\theta)}
$$

The Angle $\theta$ corresponding to the peak value of the spatial spectral function is the direction of the radial flow to be estimated. After the size and direction of the radial flow are obtained, the radial flow field diagram of the whole detected sea surface can be obtained. Fig.3 shows the radial flow diagram at 7:00 on September 25, 2017 of the south-central three stations of the bistatic radar.

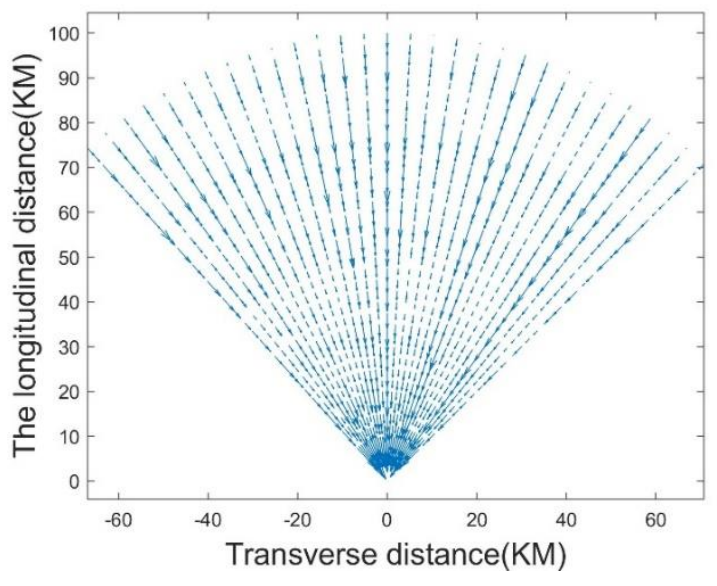

Figure 3. vector synthesis schematic.

\subsection{Synthesis of vector flow}

In double station radar to detect the overlapping area according to the longitude and latitude to construct the public grid, respectively of radial flow with the same session each stance radar site contains a single point of the radial velocity information is distributed to the corresponding mesh point, so that each public grid point contains two messages from different position of the radial velocity[7]. According to the two radial flow information, a vector flow information is synthesized to obtain the vector velocity information at the point of the common grid.

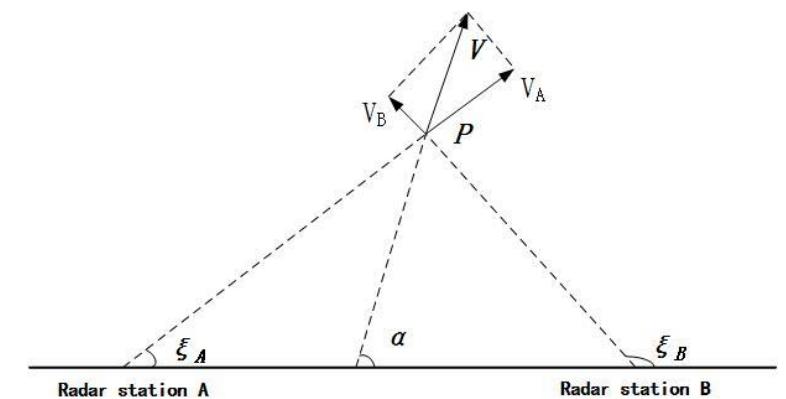

Figure 4. vector synthesis schematic.

The relationship between the direction and velocity of the vector flow and the direction and magnitude of the radial flow is shown in Fig.4. $V, \alpha$ are the velocity and di-rection of the measured vector point $\mathrm{P} ; V_{A}, \xi_{A}$ are the radial velocity and direction at $\mathrm{P}$ measured by station $\mathrm{A} ; V_{B}, \xi_{B}$ are the radial velocity and direction at $\mathrm{P}$ measured by station $B$. After the radial flow of a single station is obtained, the final velocity $V$ and flow direction $\alpha$ can be obtained by the following formula:

$$
\begin{gathered}
V_{A}=V \cos \left(a-\xi_{A}\right) \\
V_{B}=V \cos \left(a-\xi_{B}\right) \\
a=\arctan \frac{V_{B} \cos \xi_{A}-V_{A} \cos \xi_{B}}{V_{A} \sin \xi_{B}-V_{B} \sin \xi_{A}}
\end{gathered}
$$




$$
V=\frac{V_{B}}{\cos \left(a-\xi_{B}\right)}
$$

Combined with the above vector synthesis method, the radial flow diagram of the two stations can be synthesized to obtain the vector flow diagram. Fig. 5 shows the vector flow diagram combined by two stations at 10:00 on September 25, 2017.

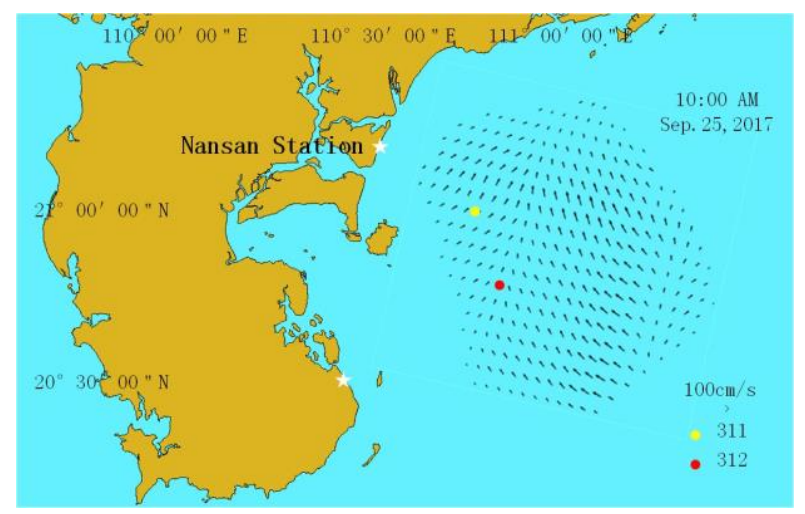

Figure 5. Composite vector flow diagram.

\section{Result Analysis}

\subsection{Comparison scheme}

In this experiment, the real-time data collected by TRDI company's DVS single-point current meter and the surface current data generated by remote sensing data were com-pared and analyzed. In general, the change of ocean surface current is slow. the bistatic compact surface-wave radar system synthesizes a vector current map every 20 minutes, and combines the four data of two single-point current meters into a group for comparison with the data of remote sensing detection.

\subsection{Result analysis}

The measured DVS and radar values from 0:00 to 12:00 on September 25, 2017 were selected for the analysis of the experimental results. Figure 6 and Figure 7 are the comparison diagram of the velocity and direction detected by the fixed-point current meter and radar at two places. It can be seen from the figure that the velocity and direction detected by radar have a high degree of agreement with the measured value. The correlation coefficients between the velocity measured by surface wave radar and the measured values of 311 and 312 were 0.8586 and 0.8475 , and the root mean square errors were $6.6 \mathrm{~cm} / \mathrm{s}$ and $6.4 \mathrm{~cm} / \mathrm{s}$, according to the results collected by current meter. The correlation coefficients between the flow direction measured by surface wave radar and the measured values of current meter 311 and 312 are 0.8844 and 0.9642 , and the root mean square errors are $24.05^{\circ}$ and $22.75^{\circ}$. The velocity and direction values detected by the compact high frequency surface wave radar system are in good agreement with those measured by the fixed-point current meter, and the overall changing trend is consistent.

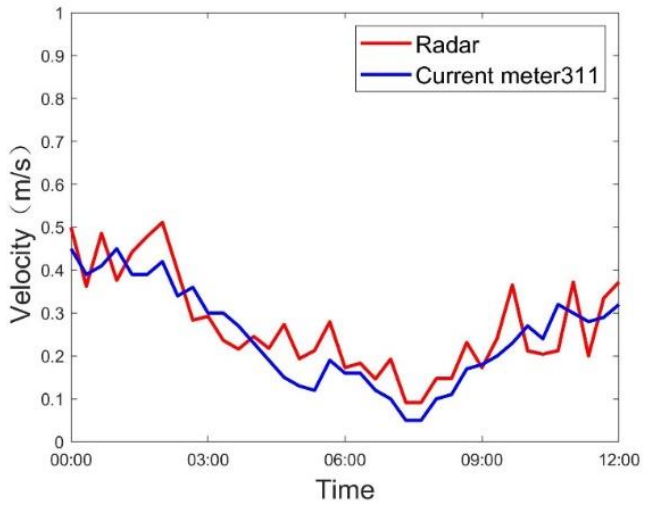

(a)

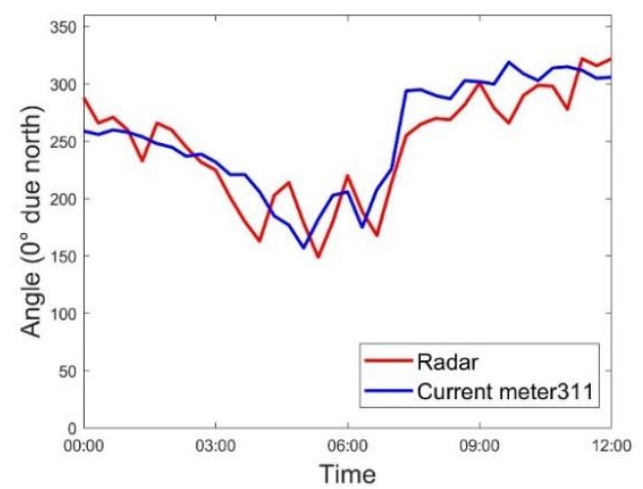

(b)

Figure 6. (a) Comparison diagram of radar data and 311 current meter rate values; (b) Comparison of radar data and 311 current meter Angle values.

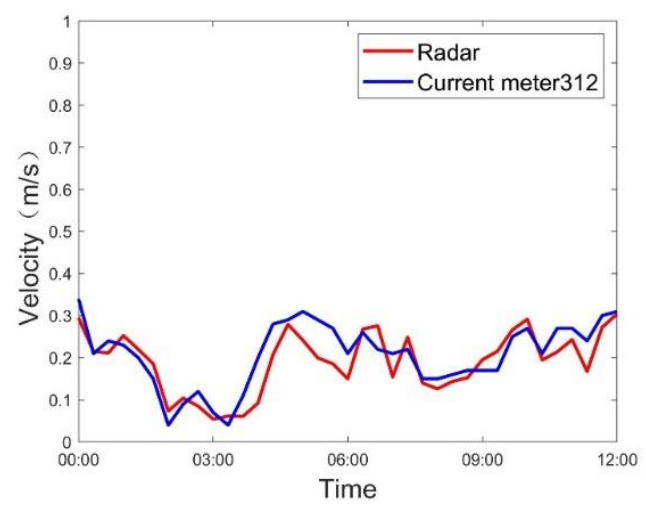

(a)

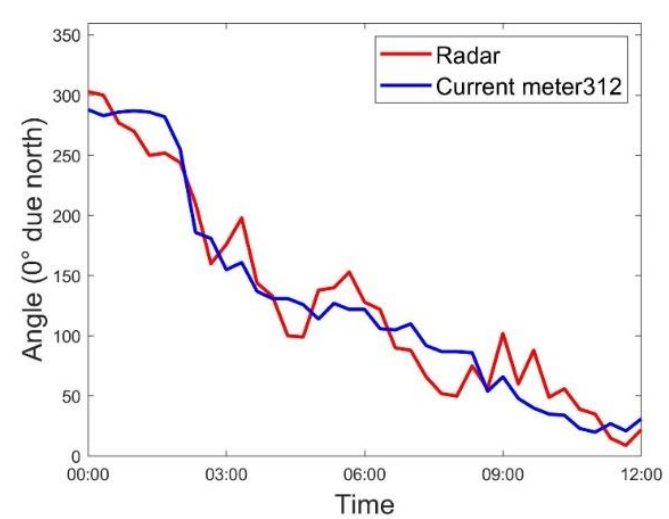

(b)

Figure 7. (a) Comparison diagram of radar data and 312 current meter rate values; (b) Comparison of radar data and 312 current meter Angle values. 


\section{Result Analysis}

The contrast experiment of surface current detection in the sea area west of Guang-dong by the compact high frequency surface wave radar adopts the ocean surface current detection equipment and the bistatic radar to make synchronous observation and com-parison in the same sea area, which fills in the blank of the previous experimental data. With the continuous development of radar detection technology, real-time monitoring of large area Marine environment has been realized, which provides a large amount of sur-face current information for intensive study of the ocean. The detection results of Omar-S in this paper are in good agreement with the observation results of other standard equip-ment, satisfy the requirement of large area real-time detection of ocean surface current, and verifying the practical performance of the compact surface-wave radar system.

\section{References}

1. X. Lingling, C. Ruixiue, S. Qingtong. Progress of Study on Coastal Circulation near the Shore of Western Guangdong. J. Journal of Guangdong Ocean University. 2012, 32, 94-98.

2. X. Xiaobai, W. Yuanqing, L. Hong.Data verification of high frequency ground wave radar and its application in typhoon monitoring. J. RADOR \& ECM. 2020, 40, 43-48.

3. Y. Hua, J. Huifeng, G. Qingqing. Summertime surface current characteristics in the southern region of Subei radial sand ridges detected by HF surface wave radar. J. Acta Oceanologica Sinica. 2019, 41, 111 .

4. W. Biyang, L. Zili, Z. Hao. Sea Surface Currents Detection at The Eastern China Sea by HF Ground Wave Radar OSMAR-S. J. Acta Electronica Sinica. 2009, 37, 2778-2782.

5. C. Jiang, W. Xiongbin, W. Biyang. Ocean Surface Current Detection by HF Ground Wave Radar with Portable Antenna System. J. Wuhan Univ. (Nat. Sci. Ed.). 2005, 51, 105-108.

6. J. Lijie, W. Biyang, Z. Hao. HFSWR with twoelement cross-loop monopole antenna array. J. Huazhong Univ. of Sci. \& Tech. (Natural Science Edition). 2017, 45, 6-10.

7. S. Fang, W. Yuanqing, L. Hong. Review of Developments and Applications Status of HighFrequency Radar. J. Hydrographic Surveying and Charting. 2018, 38, 22-30. 